\title{
Failure Mechanisms of Pressurized Microchannels, Model, and Experiments
}

\author{
M. T. Blom, Niels R. Tas, Grégory Pandraud, Emil Chmela, J. G. E. Gardeniers, Robert Tijssen, \\ Miko Elwenspoek, Associate Member, IEEE, and Albert van den Berg
}

\begin{abstract}
Microchannels were created by fusion bonding of a Pyrex cover to a thermally oxidized silicon wafer, which contained anisotropically etched grooves. Such channels are frequently used in microfluidic handling systems, for example, in chemical analysis. Since in some of these labs-on-a-chip, in particular those used in liquid chromatography, the channels are subjected to high pressures of up to a few hundred bar, it is important to have information about the mechanical stability of the channel chip, in particular of the wafer bond involved in it. The latter is the subject of this paper. The maximum pressure that can be applied to several different channel chips was investigated experimentally. In order to find the relation among this maximum pressure, channel geometry, materials elasticity, and bond energy, an energy model was developed that is generally applicable to all types of wafer bonds. It was shown that the model is substantiated by the experimental pressure data, from which it could be calculated that the effective bond energy increased from 0.018 to $0.19 \mathrm{~J} / \mathrm{m}^{2}$ for an annealing temperature ranging from 310 to $470^{\circ} \mathrm{C}$. [517]
\end{abstract}

Index Terms-Bond energy, fusion bonding, microchannels.

\section{INTRODUCTION}

W AFER fusion bonding is an important technology for microelectromechanical systems, for instance, for the fabrication of pressure sensors [1] or mechanooptical modulators [2]. Therefore, extensive research related to the technology itself and the physical mechanisms behind it has been performed [3]-[8].

For microfluidic applications, fusion bonding is important as well, although in that field anodic bonding is used more commonly [9]-[13]. For certain microfluidic applications, however, fusion bonding can still be advantageous [14], [15]. Furthermore, in some applications, the high electrical fields required in anodic bonding are undesirable, e.g., because of the risk of dielectric breakdown or charging up of some parts of the chip in question or because of the risk of bonding in unwanted areas (see Fig. 1). The latter especially will be the case in chips containing a very shallow channel or chips containing flexible parts,

Manuscript received January 5, 2000; revised May 30, 2000. Subject Editor, W. N. Sharpe, Jr. The work of M. T. Blom, N. R. Tas, G. Pandraud, J. G. E. Gardeniers, M. Elwenspoek, and A. van den Berg was supported by the Dutch Technology Foundation STW and the University of Twente. An earlier version of this paper was presented at the IEEE MEMS Conference, Miyazaki, Japan, January $23-27,2000$.

M. T. Blom, N. R. Tas, G. Pandraud, J. G. E. Gardeniers, M. Elwenspoek, and A. van den Berg are with the MESA Research Institute, University of Twente, Enschede 7500 AE, The Netherlands.

E. Chmela and R. Tijssen are with the Department of Chemical Engineering,

University of Amsterdam, the Netherlands.

Publisher Item Identifier S 1057-7157(01)01585-2.

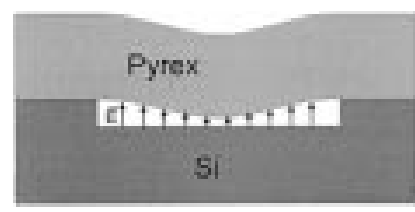

(a)

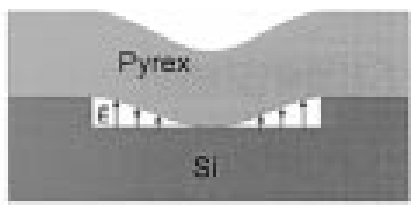

(b)
Fig. 1. An electric field across a shallow channel will induce electrostatic forces which can (a) bend and (b) even partially close the channel.

like accelerometers [16]. For such applications fusion bonding generally is a better solution.

A serious drawback of most of the fusion or direct bonding methods known today is that they require, after a room-temperature prebond, a high-temperature annealing step at $1000{ }^{\circ} \mathrm{C}$ or higher, to increase the bond strength [8]. High temperatures are undesirable in many applications, and therefore significant effort has been spent on developing bonding schemes, which require lower temperatures. Chemical mechanical polishing [8] gave an essential improvement of the quality of wafer bonds and helped in the development of low-temperature bonding procedures [8]. The question remains whether such low temperature bonding procedures lead to adequate bonding strengths.

There are only a limited number of techniques that are used for characterizing the bond strength. For direct bonding, the double cantilever beam method, also known as the crack opening method, is used. A thin blade is inserted into the bonding interface, generating a crack. The crack length is a measure for the bond strength. A disadvantage of this technique, besides its difficult reproducibility, is that it can only be used up to a certain bond strength [17]. A nondestructive technique that does not have this limitation is described in [18] and [19]. The bond strength is characterized by observing the bonding of gaps differing in size, form, and depth. This, however, only gives a qualitative measure of the bond strength.

In this paper, we present a new quantitative method to determine the effective bond energy. Although the method is applied here to fusion bonding of glass to oxidized silicon, it is generally applicable to all types of bonds.

We focus on the failure mechanisms of pressurized microchannels, thus looking at the bond strength from a microfluidic point of view. Because microchannels often are fabricated by bonding two wafers, the maximum pressure is directly related to the failure of either the bond or of one of the wafers. We consider a microchannel fabricated by bonding of a silicon and a Pyrex wafer. As Pyrex is by far the weakest material of the two: there are two failure mechanisms for a pressurized microchannel (see Fig. 2). One mechanism is cracking of the Pyrex right at the edge of the channel, as 


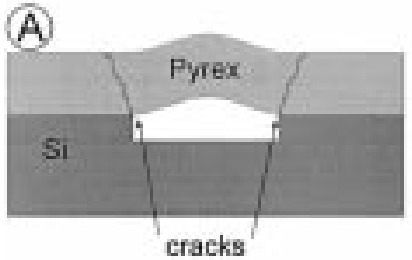

(a)

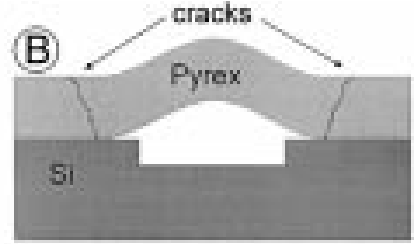

(b)
Fig. 2. Channel failure mechanisms. With mechanism A, only the Pyrex breaks. Mechanism B implies that first the bond interface opens before the Pyrex cracks.

depicted in Fig. 2(a): the sum of bending and shear stresses exceeds the maximum stress the Pyrex can withstand, and the Pyrex breaks.

The second mechanism is shown in Fig. 2(b). If the pressure is large enough, the bonding interface will open. Once that happens, the pressure acts on an increased area and the interface will open further. During this process, the stress in the Pyrex will increase until it exceeds the maximum stress at some point in the material. Depending on the geometry, the material properties, and the bond strength involved, either the glass wafer breaks first, due to mechanism A, or the Pyrex wafer peels off (B). In the latter case, information about the bond strength can be extracted. Therefore, we have focused in this work on failure mode B. A theory relating geometry, material properties, maximum pressure, and bond strength was developed. This approach is comparable to the so-called blister test for thin layers [20], [21]. However, in that case, the flow rate is kept constant, while in our situation a constant pressure is applied.

In our model, the bond strength is characterized by the effective bonding energy, which is defined as the separation energy per unit apparent contact area. The apparent contact area is the geometrical bonded area. For rough surfaces containing a certain density of asperities of different lengths, the effective bond energy $\Delta \gamma_{b}$ is lower than the work of adhesion $\Delta \gamma$ [22], [23] because 1) the real contact area is smaller than $100 \%$ and 2) part of the work of adhesion is stored as elastic deformation energy needed to compress the higher asperities in contact. The work of adhesion is defined as the work needed to separate the contacting surfaces from full contact to infinity.

By applying the energy model to bonded samples that were annealed at different temperatures, the dependence of the bond energy on the annealing temperature could be investigated.

\section{THEORY: ENERGY DESCRIPTION}

The theory relating channel pressure, geometry, material properties, and bond energy requires an energy balance. The total (free) energy for the situation of interest equals the sum of the elastic deformation energy, the surface energy, and the hydraulic energy of the pressure source

$$
U_{\text {tot }}(x, y)=U_{\text {elast }}(x, y)+U_{\text {surf }}(x, y)-p \Delta V(x, y) .
$$

where

$\Delta V$ volume change due to deflection of the Pyrex wafer;

$p \quad$ applied pressure;

$x \quad$ detachment length;

$y \quad$ channel center deflection (Fig. 3).

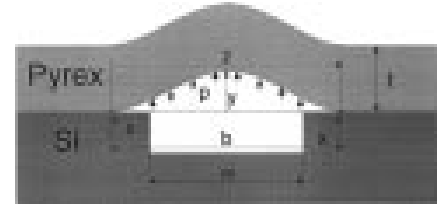

Fig. 3. Channel geometry. The distance across which the Pyrex is deflected $b$ is related to the detachment length $x$ and the actual channel width $w$ as $b=$ $2 x+w$. Also shown are the channel center deflection $y$, the pressure $p$, and the distance across the channel from one detached side called $z$.

A stable equilibrium is obtained for a minimum in the total energy for variation of $x$ and $y$, which gives the situation we want to describe. This equation is equivalent to the so-called Griffith energy balance [24], which is known from fracture mechanics.

\section{A. Elastic Deformation Energy Per Unit Channel Length}

We start the calculation of the bending energy of the Pyrex by considering the cross-section of the channel as a beam that is rigidly clamped on both sides and loaded with a uniform pressure $p$ (Fig. 3).

For a beam of length $b$, modulus of elasticity $E$, and moment of inertia $I$, the elastic deformation energy per unit channel length $L$ is given by [25, p. 492]

$$
U_{\text {elast }}=\frac{E I}{2 L} \int_{0}^{b}\left(\frac{d^{2} v(z)}{d z^{2}}\right)^{2} d z
$$

in which $v(z)$ is the deflection of the beam at a position $z$ along the beam and $b$ the distance across which the Pyrex is deflected (Fig. 3). The deformation $v(z)$ of a beam rigidly clamped on both sides and loaded with uniform pressure $p^{\prime}=p \times$ beam width $=p \times$ channel length is given by $[25, \mathrm{p} .566]$

$$
v(z)=\frac{p^{\prime} z^{2}(b-z)^{2}}{24 E I} \text {. }
$$

We have to express this in the internal variable $y$. This can be done by identifying that the maximum deflection $v_{\max }=y$ occurs at $z=1 / 2 b$. Thus $v(z)$ can be written as a function of $y$

$$
v(z)=\frac{16 y z^{2}}{b^{4}}\left(b^{2}-2 b z+z^{2}\right) \text {. }
$$

With the moment of inertia $I=1 / 12 L t^{3}$, where $t$ is the thickness of the Pyrex wafer, it follows that

$$
\begin{aligned}
U_{\text {elast }}(x, y) & =\frac{E I}{2 L} \\
\int_{0}^{b}\left(\frac{d^{2} v(z)}{d z^{2}}\right)^{2} d z & =\frac{128}{15} \frac{E t^{3}}{b^{3}} y^{2} .
\end{aligned}
$$

\section{B. Surface Energy Per Unit Channel Length}

The energy per unit channel length needed to open the bonding interface by a distance $x$ (on both sides) is given by the effective bond energy $\Delta \gamma_{b}$ multiplied with the exposed area $2 x$

$$
E_{\text {surf }}(x, y)=2 x \Delta \gamma_{b} \text {. }
$$

\section{Hydraulic Energy Per Unit Channel Length}

The volume change determining the hydraulic energy can be calculated by integrating (4) along the beam and multiplying with the channel length $L$. The hydraulic energy per unit channel length is then

$$
U_{\text {hydraulic }}(x, y)=p \Delta V(x, y)=\frac{8}{15} p y b .
$$




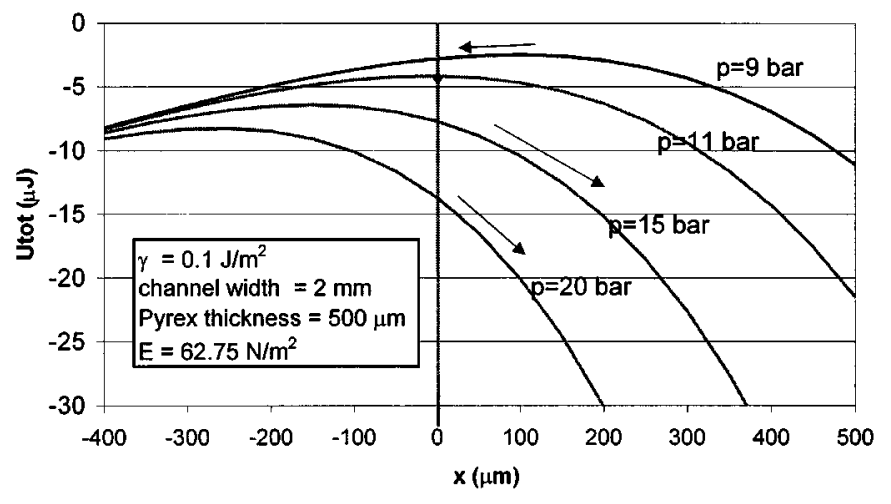

Fig. 4. Total energy as a function of the detachment length. It is clear that for pressures below 11 bar a local equilibrium exists because $x \geq 0$. For higher pressures, no equilibrium exists; therefore $x$ must increase and the bond opens.

\section{Total Energy}

For a stable situation, (1) for the total energy $U_{\text {tot }}(x, y)$ should be minimized for variation of $x$ and $y$. For $y$, this means

$$
\frac{\partial U_{\text {tot }}(x, y)}{\partial y}=0 .
$$

From this, an expression for the channel center deflection $y$ as a function of geometry, material constants, and the pressure can be derived directly. The result corresponds to [25].

Substituting this channel center deflection $y$ in the expression for the total energy gives $U_{\text {tot }}(x)$. The resulting total energy $U_{\text {tot }}(x)$ as a function of the detachment length $x$ (Fig. 3) is shown in Fig. 4 for different pressures.

Initially, $x=0$ (refer to Fig. 4). For a relatively low pressure $p=9 \mathrm{bar}$, a local energy minimum is obtained at $x=0$ because the function is bound by the condition that $x \geq 0$. At $p=11$ bar, the situation is on the verge of becoming unstable. For a further increase in the pressure, no minimum in the total energy can be found for $x \geq 0$. This results in an increase of $x$, implying that the bond interface is opened.

From Fig. 4 it can be concluded that the point where the interface starts to open can be described by $x=0$ and

$$
\frac{\partial U_{\text {tot }}(x, y)}{\partial x}=0 .
$$

Combining (8) and (9) and substituting $x=0$ gives an expression from which the pressure $p$ can be written as a function of geometry, material parameters, and effective bond energy

$$
p=\sqrt{\frac{2048 E t^{3} \Delta \gamma_{b}}{77}} \frac{1}{w^{2}}
$$

in which $w$ is the actual channel width (Fig. 3). Thus, according to this model, the critical pressure increases linearly with $1 / w^{2}$. In a plot of the maximum pressures against the reciprocal of the squared channel width, the effective bond energy can be extracted from the slope. For individual measurements, the expression above can be rewritten for the effective bond energy as a function of channel width and pressure

$$
\Delta \gamma_{b}=\frac{77}{2048} \frac{w^{4} p^{2}}{E t^{3}}
$$

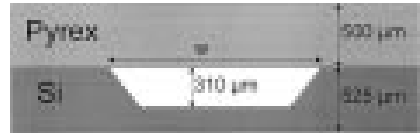

Fig. 5. Channel cross-section. The silicon is patterned using $\mathrm{KOH}$ etching leaving an anisotropic etch profile.

\section{FABRICATION AND ROUGHNESS CHARACTERIZATION OF THE TEST STRUCTURES}

All process steps except for the annealing were done in a cleanroom environment. Principally, the channels were etched into a 4-in silicon wafer and closed by fusion bonding a 3-in Pyrex wafer on top.

At first, a 160-nm low-stress silicon nitride layer was deposited on a 4-in (100) silicon wafer and patterned with reactive ion etching using a $\mathrm{CHF}_{3} / \mathrm{O}_{2}$ mixture. Subsequently, the wafers were etched in a $25 \mathrm{wt} \% \mathrm{KOH}$ solution at $75^{\circ} \mathrm{C}$ for $302 \mathrm{~min}$, giving an etch depth of approximately $310 \mu \mathrm{m}$. After cleaning and stripping the nitride mask layer, a $1-\mu \mathrm{m}$ silicon oxide layer was grown by wet thermal oxidation at $1150{ }^{\circ} \mathrm{C}$. Thus the silicon wafer and the Pyrex wafer were hydrophilic.

The Pyrex wafer was polished for 5 min using a Semi Sperse $25^{1}$ polishing solution. After Piranha cleaning $\left(\mathrm{H}_{2} \mathrm{SO}_{4}: \mathrm{H}_{2} \mathrm{O}_{2}=\right.$ 1 : 3 for $20 \mathrm{~min}$ at $100{ }^{\circ} \mathrm{C}$ ), rinsing and dry-spinning both the silicon and the Pyrex wafer, they were contacted and a prebond was formed. This prebond could be enhanced somewhat by using a pair of tweezers to remove air bubbles on the side of the wafer pair.

Four wafer pairs were annealed for $2 \mathrm{~h}$ at different temperatures: $316^{\circ} \mathrm{C}, 363{ }^{\circ} \mathrm{C}, 425^{\circ} \mathrm{C}$, and $470{ }^{\circ} \mathrm{C}$. The annealing temperature is limited for practical reasons by the strain point of the Pyrex, which is $510^{\circ} \mathrm{C}$ according to the manufacturer's ${ }^{2}$ specification.

The channel height given by the $\mathrm{KOH}$ etch depth is designed for the use of common capillaries with an outer diameter ranging from 260 to $300 \mu \mathrm{m}$. This gives a channel cross-section, as shown in Fig. 5.

The channel ends are adapted for use of the same capillaries as mentioned above. Therefore, they are $560 \mu \mathrm{m}$ wide for a 6-mm distance on both sides ( Fig. 6). On a 4-in wafer, only five channels were created in order to leave sufficient bonding area. The channel widths are 560, 750, 1000, 1500, and 2000 $\mu \mathrm{m}$. The channels are linked by a thin $(20 \mu \mathrm{m}$ wide $)$ channel to the outer world. This is done in order to release the pressure that would build up during annealing in the otherwise closed channels. The resulting mask structure is shown in Fig. 6.

By dicing along the thin channel, the channels are opened. Before testing, capillaries were glued into both channel ends, using epoxy glue cured at $100^{\circ} \mathrm{C}$.

Prior to bonding, the glass interface was characterized by atomic force microscopy (AFM) measurements (Fig. 7). This gives the possibility of estimating the real contact area in the bonded area. The roughness, elastic, and adhesion parameters have been collected in Table I.

\footnotetext{
${ }^{1}$ Cabot Microelectronics, Aurora, CA.

${ }^{2}$ Corning, New York.
} 


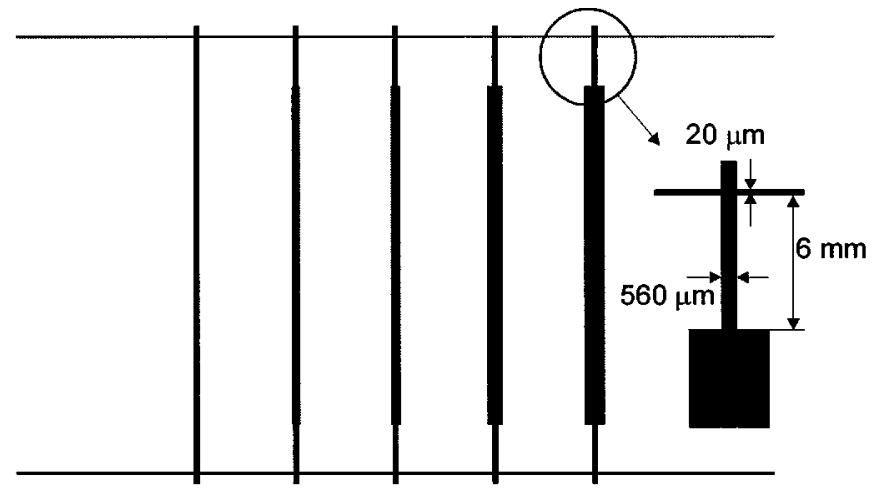

Fig. 6. Mask structure. Five channels having different widths are made. The channel ends are adapted to facilitate insertion of glass capillaries.

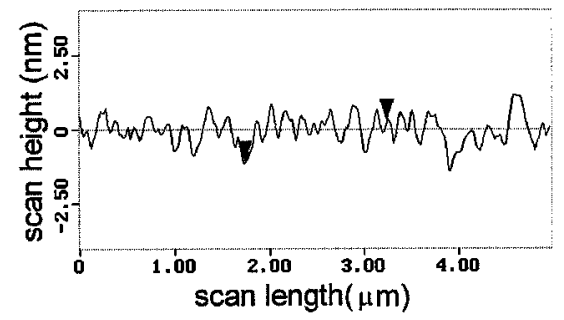

Fig. 7. Cross-section of a Pyrex AFM scan, from which the adhesion parameter can be calculated.

TABLE I

Roughness, Elastic, AND ADHESION PARAMETERS OF THE CONTACTING SURFACES

\begin{tabular}{l|l|l}
\hline Asperity Density & $\eta$ & $4 \times 10^{13} \mathrm{~m}^{-2}$ \\
\hline Asperity Radius & $\mathrm{R}$ & $2 \mu \mathrm{m}$ \\
\hline Work of Adhesion & $\Delta y$ & $0.1 \mathrm{~J} \cdot \mathrm{m}^{-2}$ \\
\hline SD Summit Level & $\sigma$ & $0.4 \mathrm{~nm}$ \\
\hline Compliance & $\mathrm{D}$ & $1.59 \times 10^{-11} \mathrm{~Pa}^{-1}$ \\
\hline RMS Roughness & $\sigma_{\mathrm{RMS}}$ & $0.5 \mathrm{~nm}$ \\
\hline
\end{tabular}

Using an elastic adhesive contact model [22], [23], the normalized real contact area as a function of the dimensionless adhesion parameter $\theta$ can be found (Fig. 8). This model is valid only under the assumption that the deformation is elastic. This can be checked by calculating the maximum pressure resulting from the Hertzian contact $q_{0}$

$$
q_{0}=\sqrt{\frac{\frac{9}{4} Z}{\pi^{2} D^{2} R}}
$$

in which

$Z \quad$ identation of the asperity;

$D$ compliance;

$R \quad$ asperity radius.

The values for $R$ and $D$ are given in Table I. The indentation of the highest asperities is approximately $1-2 \mathrm{~nm}$ giving a value of $q_{0}=1.1 \mathrm{GPa}$. From the Pyrex hardness $K=4.1 \mathrm{GPa},{ }^{3}$ it can be concluded that the assumption of elastic contact is justified. The normalized real contact area $A^{*}$ is defined by [26], [27]

$$
A^{*}=\frac{A_{r}}{\eta R \sigma A_{a}} .
$$

${ }^{3}$ See http://www.quartztecheng.com/products.htm.

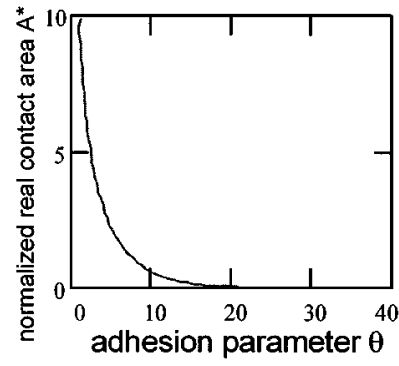

Fig. 8. Normalized contact area $A *$ versus the adhesion parameter $\theta$ for equilibrium with zero applied (external) load [22], [23].

with $A_{r}$ the real contact area, $A_{a}$ the apparent contact area, $\eta$ the asperity density, and $\sigma$ the standard deviation of the summit level. Using the measured parameters, $A^{*}=30$ corresponds to full contact. The adhesion parameter is defined by [28]

$$
\theta=\frac{\sigma^{3 / 2} R^{1 / 2}}{D \Delta \gamma R} .
$$

After prebonding, the work of adhesion is expected to be 0.1 $\mathrm{J} / \mathrm{m}^{2}$ [5]. Assuming that the silicon wafer is perfectly smooth, and taking the parameters from Table I, it can be calculated that the adhesion parameter equals 3.6 and the relative real contact area $A_{r} / A_{a}$ is $12 \%$. The consequence is that the effective bond energy will be lower than the work of adhesion, due to both the incomplete contact and the elastic deformation of contacting asperities. Based on the elastic contact model [22], [23], an effective bond energy of $0.01 \mathrm{~J} / \mathrm{m}^{2}$ is calculated for a work of adhesion of $0.1 \mathrm{~J} / \mathrm{m}^{2}$.

\section{Pressure Test Procedure}

\section{A. Model Remarks}

From the fabricated structure shown in Fig. 5, it becomes clear that a relatively deep channel is etched into the silicon, leaving a thin silicon channel bottom.

Thus, beside the Pyrex, the silicon will bend as well during pressurization. This bending will occur mainly at the thin channel bottom, because there the bending stiffness is approximately 15 times lower than that of the unetched part of the silicon. The bending of the channel bottom in itself does not alter the calculations. However, some of the stresses caused by that bending will be transferred along the channel walls to the point where the channel walls and the Pyrex meet. This will somewhat affect the pressure at which the bond opens. Because of the thin channel bottom, this effect will be negligibly small and will therefore be neglected.

\section{B. Measurement Setup}

For the pressure measurements, a Spectroflow 400 solvent delivery system (Kratos, Germany) with a pressure range from 0 to 400 bar and a flow rate from 0 to $4.99 \mathrm{ml} / \mathrm{min}$ was used. The pump was equipped with a Bourbon pressure gauge with electrooptical converter and a three-digit display in bar.

Providing a flow to a high-resistance fluid recirculation loop resulted in a pressure drop. Splitting the loop at the high-pressure side created a static pressurized liquid source. The flow resistance consisted of fused silica capillaries (75 $\mu \mathrm{m} \mathrm{ID,} 55 \mathrm{~cm}$ long and $100 \mu \mathrm{m} \mathrm{ID,} 32 \mathrm{~cm}$ long). The pressure was varied by 


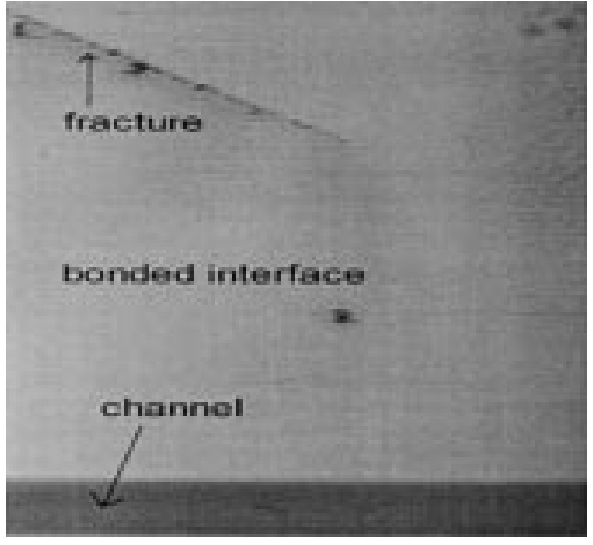

Fig. 9. Interface after pressure test (top view). It is clear that there is some distance between the onset of the crack and the channel edge. Thus the interface must have opened.

changing the flow rate in the loop. Static pressure to the chip was applied by connecting the capillaries that were glued on both sides of the channel to the high-pressure split from the source.

\section{Measurement Procedure}

For each channel, the pressure was increased in steps that depended on the expected maximum pressure. For maximum pressures that were expected from theory to lie below 15 bars, the pressure was increased by 1 bar; otherwise, the step size was 2 bars. After each step, the pressure was allowed to stabilize.

Essential for the comparison of measurements and theory is that failure of the channel occurs according to mechanism B. Thus the bond interface should crack before the glass breaks, as shown in Fig. 2(b). It turned out that for all measurements, mechanism B was indeed the leading mechanism. In Fig. 9, this becomes clear because the crack in the Pyrex starts at a certain distance from the channel edge. Evidence for the fact that the interface indeed was opened was given by fluid flowing out of the glass crack along the Pyrex-silicon interface. Consequently, the developed theory can be applied to the measurements.

\section{Measurement Accuracies}

Because of the measurement procedure described in Section IV-C, in which the pressure was raised in steps, the uncertainty in the pressure measurement will be partially due to the step size with which the pressure was increased and partially due to the inaccuracy of the pressure measurement. For each measurement point, the error was determined individually. Because pressure measurements were done for different channel widths, the error in the channel widths should be known as well. This is mainly determined by the underetch after $\mathrm{KOH}$ etching. Thus the error in channel width $w$ will be $\pm 2 \mu \mathrm{m}$.

The error in the resulting bond strength $\Delta \gamma_{b}$ can be determined from (10). The square root in that equation is the slope in a graph relating pressure $p$ and $1 / w^{2}$. Thus the relative error in bond strength is, using a first-order Taylor expansion

$$
\frac{\Delta\left(\Delta \gamma_{b}\right)}{\Delta \gamma_{b}}=2 \frac{\Delta \text { slope }}{\text { slope }}+3 \frac{\Delta t}{t}+\frac{\Delta E}{E} .
$$

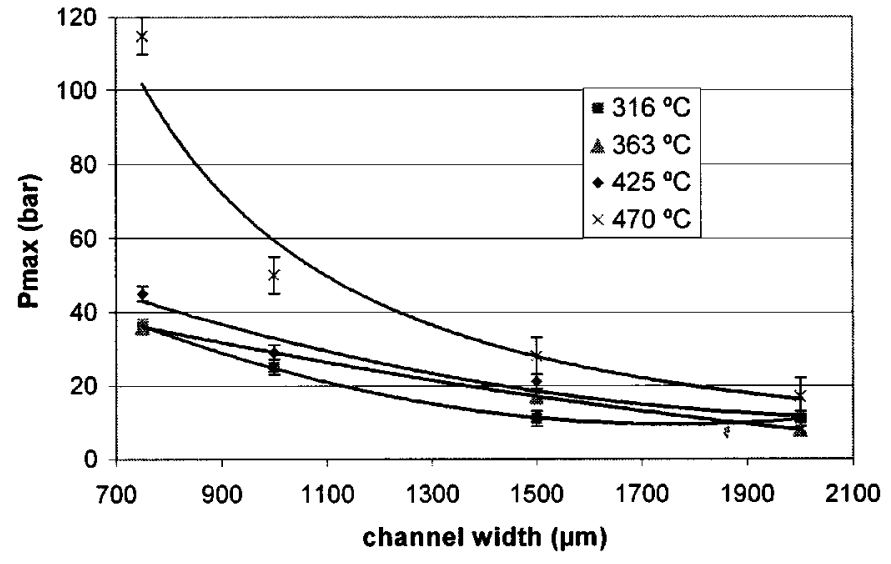

Fig. 10. Maximum pressures for different channel widths. The lines are curve fits.

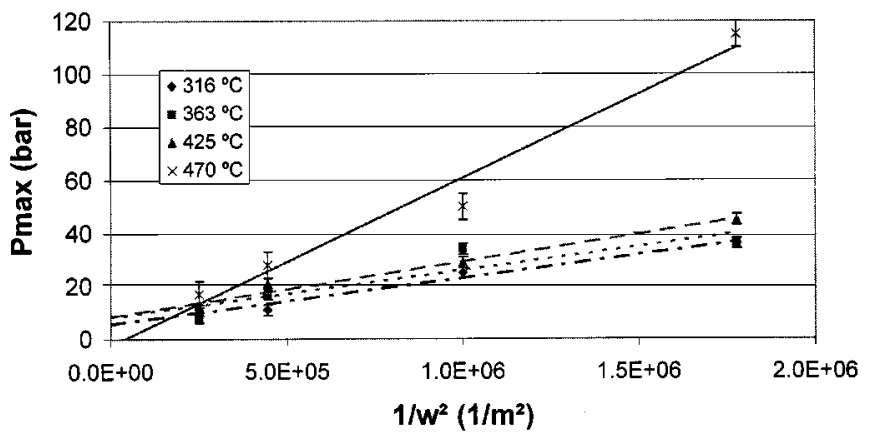

Fig. 11. Maximum pressure versus $1 / w^{2}$. It can be concluded that the predicted linear dependence of the maximum pressures on the reciprocal of the channel width squared is correct.

The thickness inaccuracy $\Delta t$ is $25 \mu \mathrm{m}$, giving a total contribution of $15 \%$ to the bond strength error. The inaccuracy in Young's modulus $E$ is negligible; thus only the uncertainty in the slope, which can be determined graphically, should be added to that.

\section{Pressure Test Results}

The results of the pressure tests are shown in Fig. 10. The collapse pressure is smallest ( 9 bar) for the widest channel (2.0 $\mathrm{mm}$ ). A graph (Fig. 11) relating the maximum pressures and the reciprocal of the channel width squared shows that the predicted linear dependence of the maximum pressure on $1 / w^{2}$ is correct, especially for lower pressures.

Deviations from the theory can be due to extra stresses in the silicon, as mentioned earlier. These, of course, will be more prominent at higher pressures, which could explain the deviations observed for such conditions in Fig. 11. Thus, for a better evaluation of the theory, thicker silicon wafers and more shallow channels should be used, so that really only the Pyrex bending is of influence. Another reason for deviations could be a nonuniform temperature distribution during annealing, resulting in local bond strength variations.

Furthermore, it can be concluded from Fig. 11 that the slope of the $p_{\max }$ versus $1 / w^{2}$ curves depends on the anneal temperature. Because the modulus of elasticity and the Pyrex thickness are assumed to be the same for all samples, this is due to 


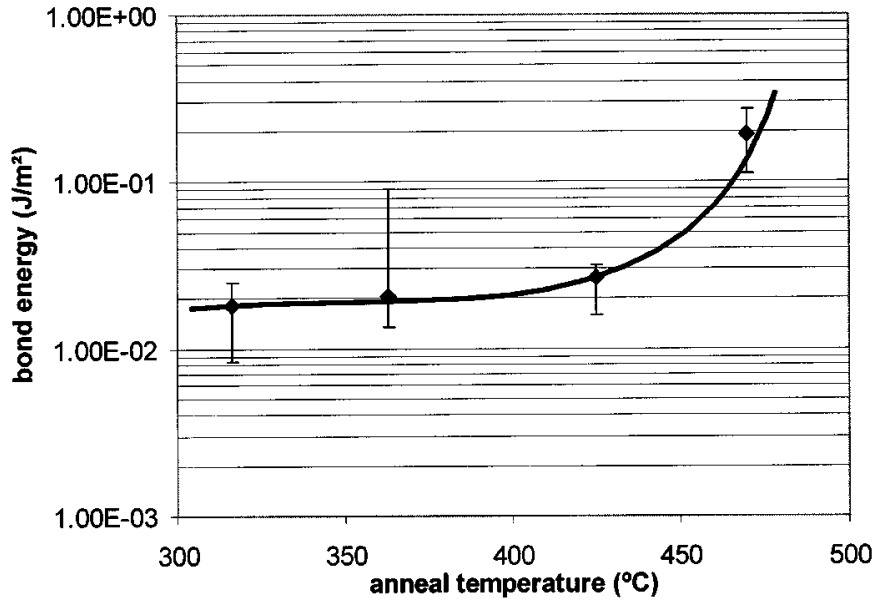

Fig. 12. Effective bond energy for different annealing temperatures. The bond energy increases with increasing temperature. The drawn line is a guide to the eye and does not represent a physical model.

a difference in bond strength according to (10). This is shown in Fig. 12. The effective bond energy increases from 0.018 to $0.19 \mathrm{~J} / \mathrm{m}^{2}$ with the anneal temperature increasing from 310 to $470{ }^{\circ} \mathrm{C}$. This increase in bond strength with temperature is explained in [3], [5], and [7] by the chemical formation of stronger bonds during annealing.

The increase of the work of adhesion during annealing will increase the effective bond energy for the area already in contact. It will also lead to an increase in the effective bond energy by growth of the real contact area.

The wafer pair annealed at $310^{\circ} \mathrm{C}$ has an effective bond energy of about a factor two higher than the calculated prebond strength. This can be explained by the fact that the increase of bond strengthening by hydrogen bond formation between silanol groups has already started at this temperature [3], [5], [7].

The effective bond energy at the lowest anneal temperature is $0.018 \pm 0.007 \mathrm{~J} / \mathrm{m}^{2}$. Using the elastic contact model and the measured roughness parameters, this implies a work of adhesion of $0.13 \pm 0.04 \mathrm{~J} / \mathrm{m}^{2}$. For the highest annealing temperature, an effective bond energy of $0.19 \pm 0.05 \mathrm{~J} / \mathrm{m}^{2}$ was found, which corresponds to a work of adhesion of $0.44 \pm 0.12 \mathrm{~J} / \mathrm{m}^{2}$.

This is in agreement with the adhesion energies given for hydrophilic-hydrophilic wafer pairs in [3].

\section{CONCLUSIONS}

For pressurized microchannels, the relation between maximum pressure, channel geometry, materials elasticity, and bond energy was investigated. An energy model was developed, which was substantiated by the measured yield pressures. The predicted linear dependence of the maximum pressures on the reciprocal of the channel width squared was found to be correct. The model was used to calculate the effective bond energy for fusion bonding of Pyrex to oxidized silicon. The effective bond energy increased from 0.018 to $0.19 \mathrm{~J} / \mathrm{m}^{2}$ for an annealing temperature increasing from 310 to $470{ }^{\circ} \mathrm{C}$. Corrected for the influence of surface roughness, this implies that the work of adhesion increased from 0.13 to $0.44 \mathrm{~J} / \mathrm{m}^{2}$.

\section{ACKNOWLEDGMENT}

The authors are grateful to M. Dijkstra for polishing the Pyrex wafers.

\section{REFERENCES}

[1] K. E. Petersen, "Silicon as a mechanical material," Proc. IEEE, vol. 70, pp. 420-457, 1982.

[2] G. J. Veldhuis, C. Gui, T. Nauta, T. M. Koster, J. W. Berenschot, P. V. Lambeck, J. G. E. Gardeniers, and M. Elwenspoek, "Mechano-optical waveguide on/off intensity switch," Opt. Lett., vol. 23, pp. 1532-1534, 1998.

[3] W. P. Maszara, G. Goetz, A. Caviglia, and J. B. McKitterick, "Bonding of silicon wafers for silicon-on-insulator," J. Appl. Phys., vol. 64, no. 10, pp. 4943-4950, 1988.

[4] J. Haisma, G. A. C. M. Spierings, U. K. P. Biermann, and A. A. van Gorkum, "Diversity and feasibility of direct bonding. A survey of a dedicated optical technology," Appl. Opt., vol. 33, pp. 1154-1169, 1994.

[5] R. Stengl, T. Tan, and U. Gösele, "A model for the silicon wafer bonding process," Jpn. J. Appl. Phys., vol. 28, no. 10, pp. 1735-1741, 1989.

[6] Y. Bäcklund, K. Hermansson, and L. Smith, "Bond-strength measruements related to silicon surface hydrophilicity," J. Electrochem Soc., vol. 139, no. 8, pp. 2299-2301, 1992.

[7] G. Kissinger and W. Kissinger, "Void-free silicon-wafer-bond strengthening in the $200-400^{\circ} \mathrm{C}$ range," Sensor Actuators A, vol. 36, pp. 149-156, 1993.

[8] Q.-Y. Tong and U. Gösele, Semiconductor Wafer Bonding. New York: Wiley, 1999.

[9] G. Ocvirk, E. Verpoorte, A. Manz, M. Grasserbauer, and H. M. Widmer, "High performance liquid chromatography partially integrated onto a silicon chip," Anal. Meth. Instrum., vol. 2, no. 2, pp. 74-82, 1995.

[10] S. Hannoe, I. Sugimoto, and T. Katoh, "Silicon-micromachined separation columns coated with amino acid films for an integrated on-chip gas chromatograph," in Proc. $\mu$ TAS, Banff, Canada, 1998, pp. 145-148.

[11] B. He and F. Regnier, "Microfabricated liquid chromatography columns based on collocated monolith support structures," in Proc. $\mu$ TAS, Banff, Canada, 1998, pp. 451-455.

[12] N. J. Mourlas, D. Jaeggi, A. F. Flannery, B. L. Gray, B. P. van Drieënhuizen, C. W. Storment, N. I. Maluf, and G. T. A. Kovacs, "Novel interconnection and channel technologies for microfluidics," in Proc. $\mu$ TAS, Banff, Canada, 1998, pp. 27-30.

[13] O. Leistiko and P. F. Jensen, "Integrated bio/chemical microsystems employing optical detection: A cytometer," in Proc. $\mu$ TAS, Banff, Canada, 1998, pp. 291-294.

[14] F. Pigeon, B. Biasse, and M. Zussy, "Low-temperature Pyrex glass wafer direct bonding," Electron. Lett., vol. 31, no. 10, pp. 792-793, 1995.

[15] Z. X. Xiao, G. Y. Wu, Z. H. Li, G. B. Zhang, Y. L. Hao, and Y. Y. Wang, "Silicon-glass wafer bonding with silicon hydrophilic fusion bonding technology," Sensors Actuators A, vol. 72, pp. 46-48, 1999.

[16] K. Kwon and S. Park, "A bulk-micromachined three-axis accelerometer using silicon direct bonding technology and polysilicon layer," Sensors Actuators A, vol. 66, no. 1-3, pp. 250-255, 1998.

[17] J. Bagdahn, M. Petzold, M. Reiche, and K. Gutjahr, "Characterization of directly bonded silicon wafers by means of the double cantilever crack opening method," in Proc. 4th Int. Symp. Semicond. Wafer Bonding, vol. 97-36, Paris, France, 1998, pp. 291-298.

[18] A. Cozma and B. Puers, "Characterization of the electrostatic bonding of silicon and Pyrex glass," J. Micromech. Microeng., vol. 5, pp. 98-102, 1995.

[19] J. A. Plaza, J. Esteve, and E. Lora-Tamayo, "Non-destructive in-situ test for anodic bonding," in Proc. Eurosensors X, Leuven, Belgium, 1996, pp. 176-180.

[20] H. Dannenberg, "Measurement of adhesion by a blister method," J. Appl. Polymer Sci., vol. 5, no. 14, pp. 125-134, 1961.

[21] H. S. Jeong and R. C. White, "Variational principle of thin film adhesion,” J. Vac. Sci. Technol., vol. A11, no. 4, pp. 1373-1376, 1993.

[22] C. Gui, M. Elwenspoek, N. R. Tas, and J. G. E. Gardeniers, "The surface adhesion parameter: A measure for wafer bondability," in Proc. IEEE MEMS Workshop, Orlando, FL, Jan. 17-21, 1999, pp. 290-295.

[23] — , "The effect of surface roughness on direct wafer bonding," J. Appl. Phys., vol. 85, no. 10, pp. 7448-7454, 1999.

[24] T. L. Anderson, Fracture Mechanics: Fundamentals and Applications, 2nd ed. Boca Raton, FL: CRC Press, 1995, pp. 36-38.

[25] J. M. Gere and S. P. Timoshenko, Mechanics of Materials, 3rd SI ed. London, U.K.: Chapman \& Hall, 1991. 
[26] J. A. Greenwood and J. B. P. Williamson, "Contact of nominally flat surfaces," Proc. Roy. Soc. London, vol. A295, pp. 300-319, 1966.

[27] D. Maugis, "On the contact and adhesion of surfaces," J. Adhesion Sci. Technol., vol. 10, no. 2, pp. 161-175, 1996.

[28] K. N. Q. Fuller and D. Tabor, "The effect of surface roughness on the adhesion of elastic solids," Proc. Roy. Soc. London, vol. A345, pp. $327-342,1975$

M. T. Blom was born in 1974, in Amersfoort, the Netherlands. He received the M.Sc. degree in applied physics from the University of Twente, the Netherlands, in 1998, where he is currently pursuing the Ph.D. at the MESA Institute.

His main research interests are microtechnology and chemical analysis, with applications in the field of wafer bonding and miniaturized chemical analysis systems.

Niels R. Tas received the M.Sc. degree in electrical engineering from the University of Twente, the Netherlands, in 1994, where he is currently pursuing the $\mathrm{Ph} . \mathrm{D}$. degree.

His thesis dealt with the modeling and design of micromachined hydraulic systems. His doctoral work is on micromachined linear motors. His current research interests include transduction science, electrostatic actuators, micromechatronics, and microtribology.

Grégory Pandraud received the Ph.D. degree from the University of SaintEtienne, France, in 1998.

He then joined the University of Twente, the Netherlands, as a Postdoctoral Researcher for one year. He is currently with Bookham Technology Ltd., where he is developing integrated optical components for DWDM applications.

Emil Chmela was born in Prague, Czechoslovakia, in 1975. He received the B.S. degree in chemistry and the M.S. degree in physical chemistry from Charles University, Prague, in 1996 and 1998, respectively. He is currently pursuing the $\mathrm{Ph} . \mathrm{D}$. degree at the Department of Chemical Engineering, University of Amsterdam, the Netherlands.

His thesis work was on development of capillary electrochromatography in solutions of linear charged polymers. His doctoral research is on the development of a micromachined integrated analytical system for polymer characterization.

J. G. E. Gardeniers was born in 1960, in Valkenburg aan de Geul, the Netherlands. He received the B.Sc., and M.Sc. degrees in chemistry and the Ph.D. degree in physics from the University of Nijmegen, the Netherlands, in 1982, and 1985, and 1990, respectively.

In 1990, he joined the Department of Electrical Engineering, University of Twente, the Netherlands, as an Assistant Professor. His main research interests are micromachining and thin-film deposition, with applications in the field of miniaturized chemical analysis and synthesis systems.
Robert Tijssen was born in 1944 in applebeck, Germany. He received the Ph. D. degree in analytical chemistry from the Technical University Delft (TUD), the Netherlands, in 1979.

He joined the Koninklikje Shell Laboratorium (KSLA, presently SRTCA), Amsterdam, the Netherlands, as a Research Chemist/Physicist in 1975, where he was later a Workgroupleader of the Analytical Group section and worked in the field of physical separation and organic chemical analysis. He was invited staff-lecturer at NATO-Advanced Study Institute, Ferrara, Italy, in 1990. Since 1993, he is part-time Professor in analytical chemistry with a specialization in separation of micromolecules, at the University of Amsterdam, the Netherlands.

Dr. Tijssen was awarded one of the first Silver Jubilee Medals in 1982. He has several Editorial Board memberships and has been Secretary (since 1990) and Chairman (since 1994), of the Workgroup Separation Methods of the Royal Dutch Chemical Society (KNCV).

Miko Elwenspoek (M'94-A'95) was born in 1948, in Eutin, Germany. He studied physics at the Free University of (West) Berlin. He received the Ph.D. degree from the Freie Universität Berlin in 1983.

His master's thesis dealt with Rayleigh scattering from liquid glycerol using light coming from a Mössbauer source. From 1977 to 1979, he worked on lipid double layers. His doctoral work was on relaxation measurements on liquid metals and alloys, in particular alkali metal alloys. In 1983, he moved to Nijmegen, the Netherlands, to study crystal growth of organic crystals in the group of Prof. Bennema of the University of Nijmegen. In 1987, he joined the University of Twente to take charge of the micromechanics group of the Sensors and Actuators lab, now called the MESA Research Institute. Since then, his research has focused on microelectromechanical systems, such as design and modeling of micropumps, resonant sensors, and electrostatic microactuators for microrobots. He is particularly interested in fabrication techniques such as the physical chemistry of wet chemical anisotropic etching, reactive ion etching, wafer bonding, chemical-mechanical polishing, and materials science of various thin films.. Since 1996, he has been a full Professor with the Transducer Technology group, Faculty of Electrical Engineering, University of Twente.

Albert van den Berg was born in 1957, in Zaandam, the Netherlands. He received the M.Sc. degree in applied physics and the Ph.D. degree from the University of Twente, the Netherlands, in 1983 and 1988, respectively.

His doctoral research was on chemically modified ISFETs. From 1988 to 1990, he was with the Swiss Center for Microelectronics and Microtechnology, Neuchâtel, Switzerland, as a Project Manager in the chemical sensors department. From 1990 to 1993, he conducted research on miniaturized chemical sensors and sensor systems at the IMT, University of Neuchâtel. Since 1993, he has been a Research Coordinator of Micro Total Analysis Systems ( $\mu$ TAS) at MESA, University of Twente. In 1998, he became a full Professor of miniaturized systems for (bio)chemical analysis with the Faculty of Electrical Engineering. He is a member of the $\mu$ TAS Steering Committee and Editor of the $\mu$ TAS section of Sensors and Actuators B. His current research interests focus on theory, technologies, new devices, and applications of micro- and nanofluidics for miniaturized (bio)chemical synthesis and analysis systems. 